\title{
Close Proximity Operations for Implementing Mitigation Strategies*
}

\author{
D.J. Scheeres ${ }^{\dagger}$
}

\begin{abstract}
Central to almost any characterization or mitigation mission to a small solar system body, such as an asteroid or comet, is a phase of close proximity operations on or about about that body for some length of time. This is an extremely challenging environment in which to operate a spacecraft, surface vehicle, or human mission. Reasons for this include the a priori uncertainty of the physical characteristics of a small body prior to rendezvous, the large range that can be expected in these characteristics, and the strongly unstable and chaotic dynamics of vehicle motion in these force environments. To succesfully carry out close proximity operations about these bodies requires an understanding of the orbital dynamics close to them, a knowledge of the physical properties of the body and the spacecraft, and an appropriate level of technological sensing and control capability onboard the spacecraft. To go the next step and implement some mitigation strategy can involve even more challenges, such as placing large structures or devices on the surface of the body, inside the body, or in close proximity to the body for extended periods of time. This paper will discuss the range of possible dynamical environments that can occur at small bodies, their implications for spacecraft control and design, and technological solutions and challenges to the problem of operating on and in close proximity to the surface of these small bodies.
\end{abstract}

\section{Introduction}

Mitigation and detailed characterization of asteroids and comets requires some period of close proximity operations about them. To support close proximity operations requires an understanding of dynamics of natural material on and about small bodies, and the dynamics, navigation, and control of artificial objects on and about small bodies. In this paper we discuss some of the issues associated with close proximity operations, and draw connections between these and the design of spacecraft and mission concepts to carry out close proximity operations. A more thorough review, with an emphasis on scientific missions to small bodies, is given in. ${ }^{22}$

The strongest argument for why the design of space missions to small asteroids is challenging can best be expressed through the following chain of facts.

1. Small bodies have large ranges in crucial physical parameters.

2. Among this range of small body parameters, each set can have close proximity dynamics that are difficult in and of themselves.

3. Spacecraft designs and mission operation concepts can be driven in very different directions as a function of this close proximity dynamical environment.

4. Crucial small body parameters may not be known prior to rendezvous.

*Paper AIAA-2004-1445

${ }^{\dagger}$ Associate Professor, Department of Aerospace Engineering, The University of Michigan, Associate Fellow AIAA, scheeres@umich.edu
5. It is likely that vehicle designs and operations concepts that fit one small body may not fit another.

It is important to note that a close coupling exists between the dynamics of natural material and artificial bodies about asteroids and comets. In recent years an appreciable body of literature has been built up in both of these areas. ${ }^{22,29}$

\section{Case Study Comparison: NEAR and Hayabusa}

The difficulty in predicting and designing from the close proximity environment is perhaps best exemplified by a comparison of two missions to asteroids: one past, NASA's NEAR mission to Eros, and one on-going, the Hayabusa mission from Japan (formerly known as Muses-C).

The asteroid mission phase for the NEAR mission at Eros looks very different than the asteroid mission phase of the Hayabusa mission to asteroid Itokawa. For NEAR, there was no choice but to use an orbital approach, due to the large mass of Eros. However, due to its shape, rotation state, and rotation pole orientation, the orbital mission had to be designed carefully to avoid destabilizing interactions with the asteroid's gravity field. For Hayabusa, due to the possible low mass of its target asteroid and the large mass to area ratio of the spacecraft, it is not even guaranteed that an orbital mission is possible. Thus, the entire mission consists of forcing the spacecraft to "hover" on the sunside of the asteroid (discussed later in this paper), with an associated cost in terms of fuel and ability to measure the asteroid's gravity field. A crucial observation is that the placement of instruments on the spacecraft bus are fundamentally different for each mission. The 
NEAR mission plan required instrument boresights to be placed orthogonal to the solar array normals, while the Hayabusa mission plan requires the boresights to be anti-parallel to the solar array normals, a fundamentally different spacecraft design dictated by the spacecraft dynamics about the small body, not dictated by an abstract "design philosophy".

If relatively nothing is known about the target body, it is clear that this can lead to increased design costs and impacts during the construction phase of the spacecraft as it will be necessary to design a spacecraft that covers a wider range of possible orbit and close proximity strategies. This will invariably lead to higher design and fabrication costs and to increased spacecraft mass. This places a strong driver on discovering as much as possible about the physical characteristics of potential target bodies prior to spacecraft and missin design.

Again, let us consider the Hayabusa and NEAR spacecraft designs. The Hayabusa design is appropriate for very small bodies, and is designed to maximize scientific return from visiting such bodies. On the other hand, if the Hayabusa spacecraft were sent to explore Eros, it is likely that the amount and quality of scientific measurements it could take would be significantly less than the NEAR spacecraft accomplished, and would require the spacecraft operations team to work much harder to accomplish them. The NEAR spacecraft design is appropriate for larger bodies such as Eros and was operated in that environment with a relatively small operations team. If, however, the NEAR spacecraft was used to explore a sub-kilometer asteroid it would have had profound difficulties in carrying out its mission and accumulating quality scientific measurements of that body, again at the expense of stressing the spacecraft operations team.

Thus, spacecraft designs and mission scenarios that are optimized for one class of small body may not function well at all for a different class of small bodies. For missions that wish to visit multiple asteroids, this may force a difficult trade to be made between designing a spacecraft that can accomplish a mission to range of asteroid classes or designing a mission that only explores asteroids that fall within a restricted class of physical parameters.

\section{Defining the Force Environment}

Given the large range of possible situations, and the diverse types of motion that can ensue, it is crucial that the force environment of a hazardous small body be defined as early as possible, as it is much more difficult to design a mission and spacecraft to a body with unknown characteristics. This means that ground-based characterization must play an important role in initial mission and spacecraft design.

\section{Pre-Rendezvous Characterization}

There are a number of important small body parameters that are accessible using ground-based observations. Some of these measurements are routinely taken, however a number of them require targeted observations that must be planned in advance and which require additional resources. It is fortunate to note that the majority of physical parameters needed to characterize the dynamical environment of a small body can at least be constrained by ground-based observations. The list of desired physical parameters needed to define the small body force environment are: number of co-orbitals, size, density, shape, gravity field, surface morphology, spin rate and spin state, orientation of rotational angular momentum relative to orbital plane, heliocentric orbit.

The basic suite of ground-based measurements that are used to observe small bodies and which may be able to constrain some of these physical parameters are: astrometric measurements, intensity (lightcurve) measurements, spectral measurements, and range-Doppler radar imaging measurements.

\section{Post-Rendezvous Characterization}

Following rendezvous with the small body, it is necessary to develop precision models of the body using navigation data, which generally consists of radio metric tracking data, optical observations, and altimetry. The specific physical parameters needed to support close proximity operations at the small body are its: mass, gravity field, spin state, surface topography and roughness, surface gravity field and density distribution.

The process of measuring these parameters using navigation data is rather involved, and ultimately relies heavily on combining the data to perform a joint solution for all of these parameters simultaneously. One of the main difficulties in performing these solutions is generating sufficiently accurate models to use as initial estimates. This is where the presence of existing models based on ground measurements can be crucial and can significantly cut the time required to estimate these precision models. Comprehensive discussions of these procedures for asteroids are documented in ${ }^{12,13}$ and for comets in. ${ }^{11}$

The only physical model that does not arise naturally out of the navigation measurement process is the surface gravity field and internal density distribution. While the estimated gravity field (usually parameterized as a spherical harmonic expansion) contains this information, the gravity field parameterization is generally not valid at the surface of the body and the density information cannot be uniquely extracted. One way to bypass the invalidity of spherical harmonic expansions at the surface of the body is to use ellipsoidal harmonics, ${ }^{3}$ which are still formally divergent at the surface but which can be used much closer to the 
body surface. It is also possible to directly estimate ellipsoidal harmonic coefficients from existing spherical harmonic gravity field coefficients. ${ }^{2}$ In contrast, the polyhedron gravity field potentials are valid up to and even within the body, but rely on constant density assumptions. It is possible to mimic density distributions by placing mass concentrations of different density within the body, ${ }^{30}$ but this requires that the density distribution be estimated. While this is a nonunique process, a least-squares estimation technique that uses the measured shape model and gravity field coefficients as data may allow for a rational approach to estimating these distributions. ${ }^{27}$ It is important to note that the one small body for which we have an accurate gravity field and shape measurement, Eros, has only minimal density inhomogeneities. ${ }^{31}$ This provides the hope that constant density surface gravity models may be sufficient for surface operations.

\section{Close proximity dynamics and operations}

The motion of particles in close proximity to a small body will usually deviate significantly from the familiar Keplerian motion due to perturbations from solar radiation pressure, solar gravity, small body shape/gravity, and small body rotation. Due to these perturbations it is very common to find trajectories that can escape, impact, or migrate substantially over only a few orbits about the body. In Fig. 1 we show a set of three trajectories about the Eros gravity field, with only minimal perturbations to their initial conditions. We see that one orbit is stable, one escapes, and one impacts, all over a few days. Unlike most unstable and chaotic motion in the solar system, which have timescales on the order of thousands to millions of years, the time scale for these effects to act are very short, on the order of a few hours to days. Thus, these effects must be accounted for and understood in order to carry out close proximity spacecraft operations. It is important to stress that both orbital and surface motion must deal with these issues.

\section{Complicating Scenarios and Possible Resolutions}

Based on past analyses of close proximity motion, there are a number of items that can be identified as being of specific concern to the implementation of close proximity operations on or about a small body: dynamics of disturbed regoliths, orbit mechanics issues, and surface motion dynamics. Many of the complicating scenarios discussed below can be dealt with by the appropriate choice of orbiting strategy or spacecraft design. Thus, where appropriate we mention some known strategies for mitigating these adverse dynamical effects. These strategies are by no means exhaustive, but are representative of the types of approaches that can be used. Each of these strategies have their own drawbacks, making the design of a close

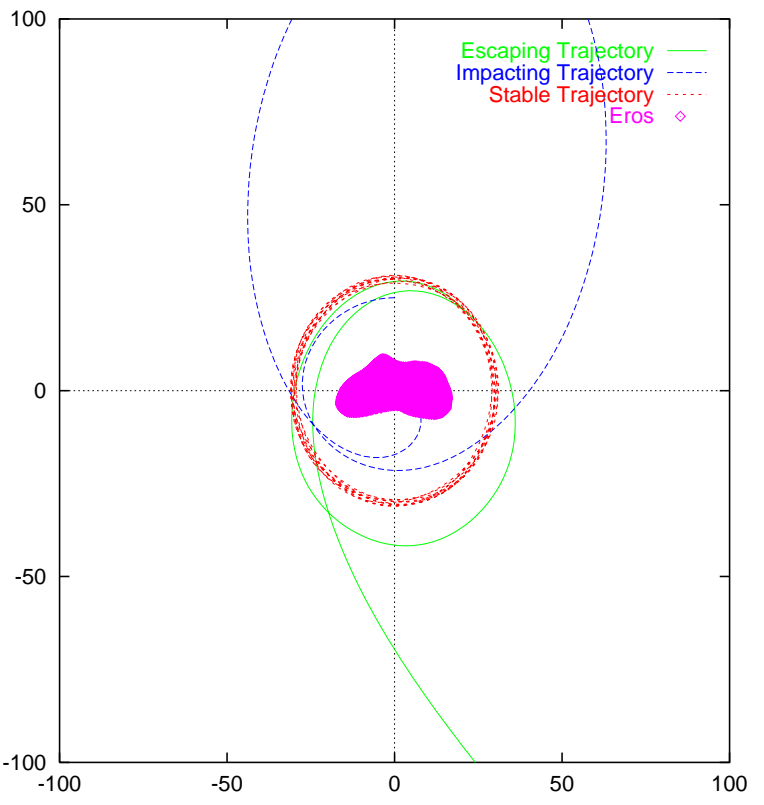

Fig. 1 Sensitivity of motion to initial conditions

proximity mission a challenging exercise in system optimization.

\section{Natural dynamics of disturbed regoliths}

An interesting idea, posed $\mathrm{in}^{24}$ in the context of exploring small body surfaces, is that operations on the surface of a small body can excite the loose regolith, effectively creating a transient atmosphere. Since escape speeds on the surface of a small body are on the order of meters per second or less, it does not require much impulse to energize regolith. If the small body were a sphere, there would only be two outcomes for each particle, escape or re-impact. However, the small body will have an irregular shape, will be spinning, and is in the solar radiation pressure and gravitational field of the sun, thus it is probable that the trajectory of non-escaping disturbed material can transition into a non-impacting orbit about the body, or at least into an orbit that will not re-impact for an extended period of time. $\operatorname{In}^{26}$ examples of this effect considering solar radiation pressure only are presented, while in ${ }^{25}$ examples of this considering the gravity field and rotation state of the small body only are presented. In both cases, re-impact of disturbed ejecta may not occur for many months! When the particles do re-impact, they will in general have speeds up to local escape speed (which is computed taking body rotation into account, see $\left.^{23}\right)$.

While this would be an interesting effect to observe, it may not be a positive environment for a landed space vehicle or a vehicle in orbit about the small body. While the "density" of the transient atmosphere would likely be very low, there may be some increased risk associated with orbiting about a body surrounded by a dusty atmosphere. Similarly, a landed vehicle could be subject to re-impacting ejecta traveling at local es- 
cape speeds, with re-impact occuring long after the initial event. More serious issues could also involve the electrostatic potential on the asteroid surface in combination with an energized regolith and the introduction of a vehicle into that environment.

\section{Orbit Mechanics}

The peculiarities of orbital motion about asteroids is a subject that is more fully understood than surface motion, and for which much recent work has been performed. For these situations the "complicating scenarios" are known to be a strong function of the body's heliocentric orbit, shape, size, rotation and density as well as the spacecraft's mass and area. The main complications are due to gravity, asteroid rotation, and solar radiation pressure effects. Each of these complications can be analyzed in isolation, but for some bodies they can play an important role in combination. We also briefly consider binary asteroids, which adds a new dimension to this problem.

Gravity and Rotational Effects First consider the effect of gravity and rotation. The simplest unifying dynamical idea for a rotating asteroid is its synchronous orbit radius, specifically the size and stability of the circular orbit that has the same period as the body's rotation. For an asteroid of a given mass, represented by its mass parameter $\mu$, and rotation period $T$, its ideal synchronous radius is computed as: $r_{s}=\left(\mu T^{2} / 4 \pi^{2}\right)^{1 / 3}$. Due to the distributed mass of the body, an asteroid will in general only have four specific locations close to $r_{s}$ where truly synchronous motion exists, ${ }^{23}$ analogous to the Earth's case, ${ }^{8}$ located along the longest and shortest body axis in the equatorial plane. For the majority of asteroids with known shapes, all four of these synchronous orbits are unstable, and trajectories started in their vicinity generally lead to impact or escape within a few orbits. This simple result lies behind most of the difficulties encountered in orbiting small bodies. In fact, for asteroids with all of their synchronous orbits unstable, motion within 2-3 synchronous radii of the asteriod mass center tends towards instability, with escaping and impacting orbits being the rule. There is a strong inclination dependence on this instability, it being the most pronounced when motion is in the plane of rotation and in the same sense (i.e., zero inclination). As motion is considered at higher inclinations, the minimum radius for stable motion tends to decrease. In Fig. 2 we show the orbital dynamics of a polar orbit about Eros. We note that the trajectory, although apparently stable initially, eventually becomes ejected from the asteroid. A similar orbit started with zero inclination would have been ejected within one or two orbits. At the other extreme, and as has been clearly established, when orbital motion is in the equatorial plane and opposite to the sense of rotation (i.e., retrograde), orbital motion is actually quite stable (see Fig.
$? ?)$

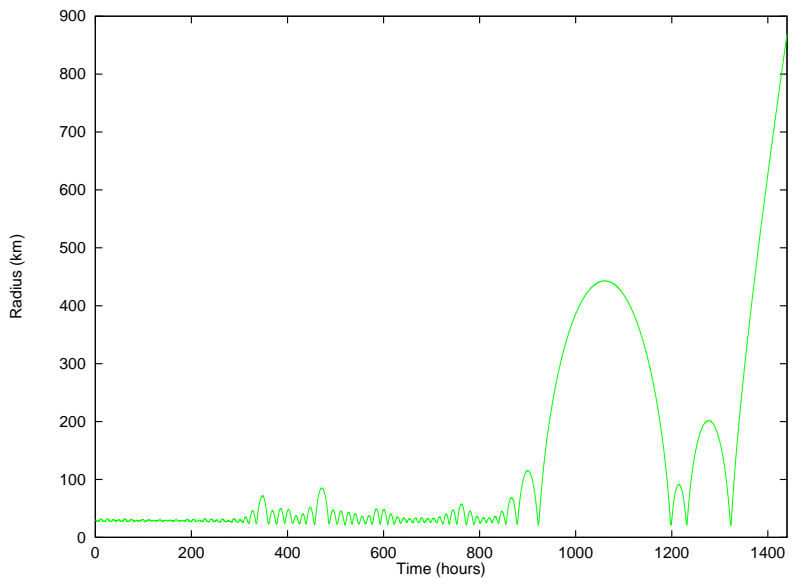

Fig. 2 Radius vs. time plot of a long-term unstable polar orbit.

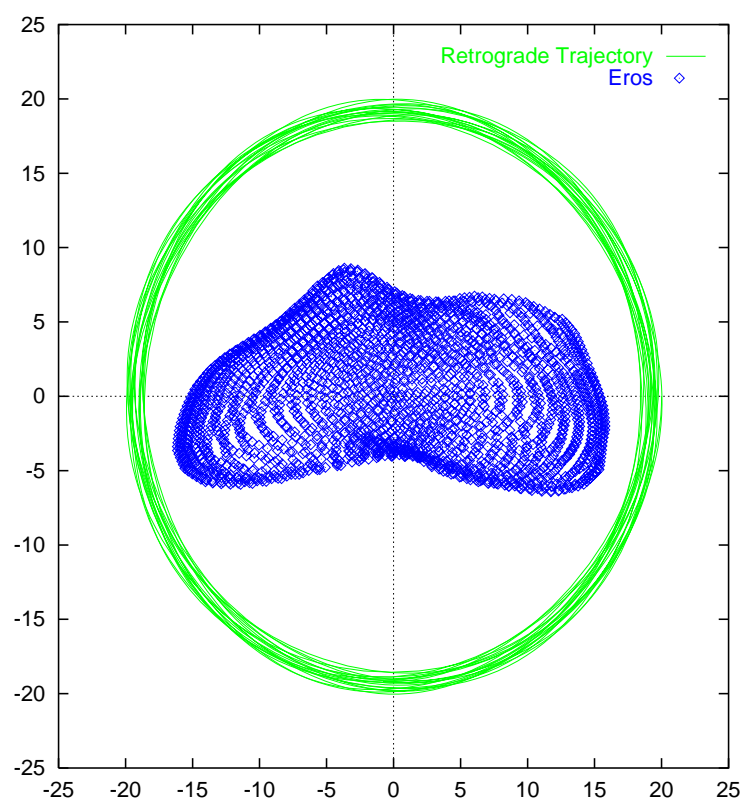

Fig. 3 Robust retrograde orbit.

The basic complication from the combined effect of gravity and rotation is that orbital motion close to the body with low relative speeds is in general not an option. Unfortunately, it is these orbits that are the most scientifically attractive for performing body-relative measurements and deploying measuring devices. The remedy is to fly in a retrograde orbit, which allows for very low altitudes relative to the long ends of the body. This, however, results in relatively high speeds with respect to the asteroid surface and places strict constraints on the geometry of close orbits.

Solar Effects While gravity and rotation work to destabilize motion close to the body, the effect of solar radiation pressure (in tandem with solar gravitational tides) is to destablize motion when relatively far from the body. The perturbative effect of solar radiation pressure combined with solar gravitational attraction is a strong function of the small body orbit, its overall 
mass, and of the spacecraft's mass to projected area ratio. Clearly, a spacecraft that strays far enough from the attracting asteroid will at some point fall under the influence of the sun. An appropriate rule of thumb for the distances at which these effects start to dominate can be approximated by the sphere of influence about the body, or more dynamically appropriately by the libration points of a particle between the asteroid and the sun. Considering only gravitational attractions, these will lie at a distance of $r_{H} \sim \pm\left(\mu / 3 \mu_{S}\right)^{1 / 3} d$ from the asteroid along the asteroid-sun line. Here, $\mu_{S}$ is the gravitational parameter of the Sun, and $d$ is the distance of the asteroid from the sun. For example, an asteroid located at $1 \mathrm{AU}$ from the sun would have its libration orbits located at $r_{H} \sim \pm 133 \rho^{1 / 3} R$ $\mathrm{km}$, where $\rho$ is the body density in $\mathrm{g} / \mathrm{cm}^{3}$, and $R$ is the mean radius of the asteroid in $\mathrm{km}$. Previous research has shown that circular orbits started with radii less than one-half of this distance are generally stable against escape from the body. ${ }^{4,5}$ Furthermore, when relatively far from the body the orbital period can be very long, leading to relatively "slow" unstable dynamics that can be easily controlled. Thus, when viewed as a gravitational perturbation alone, we see that the Sun should not stress operations or spacecraft design except for the very smallest and least dense bodies.

The inclusion of solar radiation pressure can drastically alter the situation, however. Many spacecraft are moving towards the use of solar-electric propulsion technologies, which use the sun's light to generate propulsion power. These designs naturally lead to spacecraft with relatively large solar arrays, and hence large surface areas. In addition, spacecraft are generally designed to minimize their total mass. The combined effect leads to spacecraft with relatively small projected area densitites, defined as the spacecraft mass divided by its total sun-ward projected area. For example, the Rosetta and the Hayabusa spacecraft have area densities on the order of $20-30 \mathrm{~kg} / \mathrm{m}^{2} .{ }^{28}$

The effect of the solar radiation pressure on spacecraft motion is complex, and different aspects of it are considered in, ${ }^{19,28}$ drawing on foundational work found in. ${ }^{10,15}$ Again, it is simplest to think of the effect in terms of the radius of libration points. Solar radiation pressure introduces an asymmetry between the sun-side and anti-sun-side libration points, with the sun-side point being pushed further from the asteroid and the anti-sun-side point being pushed closer to the asteroid. $\mathrm{In}^{28}$ the relative effect of the solar radiation pressure is parameterized by a quantity $\tilde{\beta}$ which is a function of the solar radiation pressure strength, the spacecraft area density, the sun's gravitational parameter, and the asteroid's gravitational parameter. Leaving out the details of the derivation we find $\tilde{\beta}=3.84 /\left(B \mu^{1 / 3}\right)$, where $B$ is the spacecraft area density in $\mathrm{kg} / \mathrm{m}^{2}$ and $\mu$ is the asteroid's gravitational parameter. When $\tilde{\beta} \leq \mathcal{O}(1)$ the sun's grav- itational effects predominate, however when $\tilde{\beta} \gg 1$ the solar radiation pressure force dominates. As an example again, the Rosetta and Hayabusa spacecraft will have estimated values on the order of $\tilde{\beta} \sim 30$. In this regime, the anti-sun-side equilibrium point is at a distance of $\sim\left(\mu / \mu_{S}\right)^{1 / 3} d / \sqrt{\tilde{\beta}}$. For a spacecraft and asteroid with $\tilde{\beta} \sim 30$ this radius shrinks to $\sim 35 \rho^{1 / 3} R$ $\mathrm{km}$, which is $25 \%$ of the gravitational only libration point distance. If $\tilde{\beta} \gg 1$ the escape dynamics of the problem also change and we find that the maximum semi-major axis that a spacecraft can have and remain captured at the asteroid is approximately one-fourth the radius of the anti-sun-ward libration point. These effects place a much more restrictive dynamical constraint on this system.

Furthermore, the dynamics themselves become much more complex, with orbital eccentricity experiencing large, periodic variations on the order of unity, which can lead to spacecraft impact after a few orbits. See Fig. 4 for plots of orbits that impact or escape from a small spherical asteroid due to solar radiation pressure, the sun is to the left. To avoid exciting such orbit eccentricity oscillations it is necessary for the spacecraft orbit plane to be perpendicular to the sun-line. Such a configuration, it turns out, yields a sun-synchronous orbit in that the solar radiation pressure will force the orbit plane to always lie perpendicular to the sun-line. See Fig. 5 for a stable orbit about the same asteroid, now the orbit plane is oriented perpendicular to the sun-direction, which is towards the left. When flying in this geometry, the spacecraft is in a terminator orbit and can have a constant eccentricity on average if its initial periapsis vector is chosen appropriately. Indeed, for small asteroids this may be the only orbital geometry that can yield feasible motion over long time spans as orbits that deviate significantly from the terminator plane may impact with the asteroid in only a few orbits. These limits and constraints can all be predicted using analytical results. ${ }^{19,26}$

A more significant issue arises when the asteroid has a non-spherical mass distribution and rotation. At the least, such mass distributions can cause precession of the orbit plane (analyzed in ${ }^{14}$ ) which in turn can excite the eccentricity oscillations which can lead to impact. If the maximum semi-major axis also happens to be within a few synchronous radii, the orbit may also be subject to destabilization from the gravity and rotation effects alone. The combination of all these effects will lead to difficult challenges for designing orbital missions to very small or under-dense bodies.

Binary Asteroids All asteroids will be subject, to some extent, to the force perturbations discussed above. Further complicating the issue, current estimates state that roughly $20 \%$ of the NEA population may be binary asteroids. While much is not known yet 


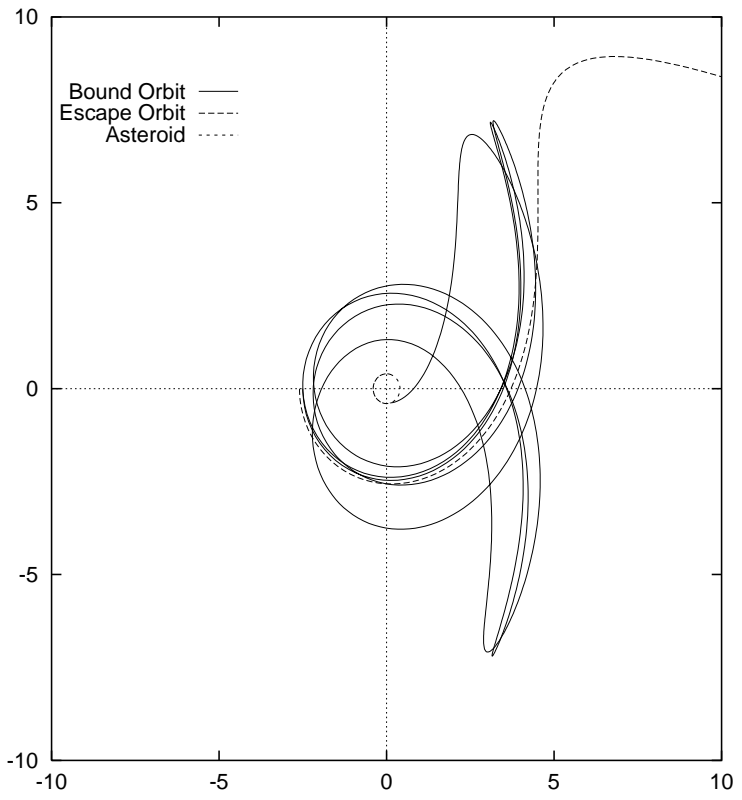

Fig. 4 Unstable orbits about a small asteroid, perturbed by solar radiation pressure only.

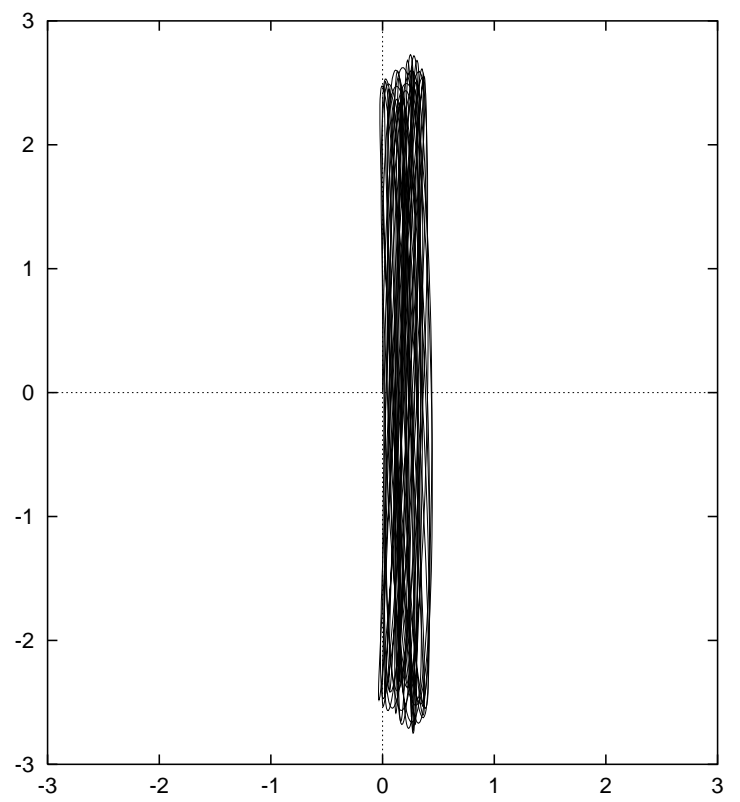

Fig. 5 Stable sun-synchronous orbit about a small asteroid, perturbed by solar radiation pressure only.

about the force environment of a binary asteroid, current indications are that it will be relatively difficult to find safe and navigable orbits close to either body. Approaching the problem from a conservative point of view, we can state that safe orbits will generally lie outside the secondary, so long as solar radiation pressure effects are not too severe, and will preferentially orbit retrograde to the binary system's orbital plane. Orbits close to or within the orbit of the secondary must contend with 3rd body forces in addition to the gravity and rotation perturbations discussed above.

One potentially attractive approach would be to place a spacecraft in one of the triangular synchronous orbits of the binary system (note, these will be synchronous with the secondary moving about the primary, but not synchronous with the rotation of the primary in general). A recent survey of known binary asteroid systems in the solar system ${ }^{21}$ shows that the majority of these systems have mass ratios small enough for the classical stability to exist. For many of these potentially stable environments, however, the gravitational disturbance of the solar tide is sufficient to destabilize their motion, such as occurs in the Earth-Moon system. If the mass ratio of the binary asteroid system is $\rho=M_{2} /\left(M_{1}+M_{2}\right)$, then the classical constraint for the triangular points to be stable is: $27 \rho(1-\rho) \leq 1$. $\operatorname{In}^{18}$ a simple criteria for when an external perturbation (such as the sun) can destabilize such a three-body system is found. Define the sidereal period of the binary system to be $T_{b}$ and the period of the binary system's orbit about the sun to be $T_{o}$. Then the condition for the triangular points to be stable when subject to the solar perturbation is: $T_{b}<27 T_{o} \rho(1-\rho) / 8$. The unstable motion associated with the solar perturbation will place a spacecraft on a potentially impacting trajectory within a few periods of the binary system. Thus, even though some NEA binaries may have stable triangular libration points, which may be a suitable place to park a spacecraft in, there will be many that have no stable solutions. Due to this, use of the triangular libration points cannot be relied upon as a generic strategy for exploring binary asteroids.

\section{Surface motion}

Finally, we can consider the motion of a vehicle (i.e., a rover) over the surface of a small body. Due to the weak gravitational attraction of the body and the uncertain properties of asteroid surfaces, the most feasible method of locomotion appears to be hopping. Several simple designs are possible, including the use of internal flywheels. ${ }^{32}$ When viewed as a means for controlled locomotion over an asteroid, however, some serious issues arise. First, let us consider some of the peculiarities of motion over the surface of an asteroid. The defining quantity for characterizing surface motion on an asteroid is the local escape speed. On the surface of a spherical, non-rotating asteroid with gravitational parameter $\mu$ and radius $R$ this speed is just $V_{e s c}=\sqrt{2 \mu / R}$. Once we consider a rotating body, however, we see that the escape speed relative to the asteroid surface will now vary with latitude and with the direction of the surface relative velocity vector. At the equator, a particle at rest on the surface will have an inertial speed of $\omega R$, where $\omega$ is the asteroid rotation rate. Surface motion in the direction of rotation at a speed of $\sqrt{2 \mu / R}-\omega R$ will be escape speed, while motion in the opposite direction of rotation at a speed larger than $\sqrt{2 \mu / R}+\omega R$ will lead to escape. A velocity 
in any other direction will have to be added (vectorially) to the velocity due to the asteroid rotation to find the total speed in inertial space, and to ascertain what this speed is relative to escape speed. Finally, if the asteroid has a non-spherical shape, we also see that the local gravitational attraction may be greater or lesser at different points on the surface of the asteroid as well as the surface speed due to rotation, leading to additional variations in escape speed. For definiteness, local escape speed is generally defined as the speed perpendicular to the surface necessary to achieve escape speed relative to the body ( $\mathrm{see}^{29}$ for a more complete definition). Such local variations can be extreme, for the asteroid Eros the local escape speed varies between 5 and $15 \mathrm{~m} / \mathrm{s}$ depending on surface location. ${ }^{13}$

Local escape speed is a useful characterization as it provides an easily computable limit on surface speeds, however we must note that speeds less than local escape speed may also end up escaping the asteroid. In $^{29}$ a classification of surface ejecta into different classes is given in terms of their final state:

Class I Immediate reimpact: ejecta reimpacts with the surface prior to first periapsis passage.

Class II Eventual reimpact: ejecta does not reimpact at the first periapsis passage, but eventually reimpacts in the future.

Class III Stable motion: ejecta is placed into a longterm stable orbit about the asteroid.

Class IV Eventual escape: ejecta has at least one periapsis passage by the asteroid before it escapes.

Class V Immediate escape: ejecta escapes from the asteroid prior to its first periapsis passage.

Local escape speed really defines Class V ejecta. For motion relative to the surface, this is a sobering thought as an apparently "safe" trajectory that does not immediately escape may enter orbit and eventually escape. For controlled surface motion, the goal should be to place a rover trajectory firmly into Class I, which will immediately re-impact with the surface. Examples of all classes can be easily found, for example in $^{25}$ a Class II trajectory that does not reimpact for almost a year is described. Such motion is clearly not suitable for controlled surface exploration using a rover.

These considerations force rover motion to be conservative, with only relatively small "hopping" speeds acceptable. This can lead to potential difficulties, however, depending on the topology of the asteroid surface. It is possible that a rover may be caught in a local potential well or gravitational basin, and that escape from this basin may require speeds large enough to place it into a Class II or higher trajectory. Being in such a situation would clearly be unacceptable, and implies that careful planning of surface motion trajectories are necessary prior to placing a rover on the surface of a small body. Specific strategies would probably involve the identification of paths that lead from regions of high potential to regions of low potential, avoiding large obstructions along the way.

A separate issue is the coupling between the reimpacting rover and the surface. Due to the complex topography, gravity, and the small body's rotation the motion of a vehicle with rebound can become quite complex. Thus, it is desired to design rovers to have minimum recoil speeds (i.e., low coefficients of restitution). A complete understanding of this issue requires better knowledge of the properties of asteroid regoliths, as these asteroid soils may have features that will minimize recoil speeds. Still, the apparent presence of exposed bedrock on some asteroids ${ }^{7}$ implies that the mechanical design of the rover will still be an important element. Further complications can arise from coupling between the rover rotational and translational motion. The energy of a reimpacting body may be partially converted into rotational kinetic energy and stored until the next impact with the asteroid surface. Such energy storage can yield complex and unpredictable motions of a body across the surface of an asteroid, and is an aspect of dynamics that is poorly understood and modeled. ${ }^{16}$

Finally, the coupling of surface motion with the disturbance and electric potential of surface regolith mentioned previously could potentially create a poor environment for the mechanical and electrical operation of a sophisticated rover vehicle. Initial designs of rover vehicles may be biased towards self-contained designs with a minimal number of sensor portals, such as the proposed MINERVA rover for the Hayabusa mission. ${ }^{32}$

\section{Active Control Strategies for Close-Proximity Dynamics}

An exciting possibility for close-proximity motion relative to small bodies is to take advantage of the relatively weak gravitational accelerations and actively control a spacecraft's motion relative to the body. Indeed, in the Hayabusa mission such strategies have already been developed at a basic level and will be used to circumvent the problems associated with trying to follow an orbital approach for that mission. ${ }^{6,9}$ Active control techniques may not always be suitable for specific bodies and specific missions, still they can form a very important class of motions that will surely be applicable to the exploration of smaller asteroidal and cometary bodies.

There are two general approaches to controlled motion: near-inertial hovering and body-fixed hovering. In near-inertial hovering the spacecraft is stationed at a fixed location relative to the asteroid in the sun-asteroid frame, the asteroid rotating beneath the 
spacecraft. Such a situation is useful for the initial characterization and measurement of the body. In body-fixed hovering the spacecraft is stationed at a fixed location relative to the rotating asteroid, implying that the spacecraft is rotating with the asteroid in inertial space. This situation is useful for sampling a small-body surface or for motion across the surface of an asteroid. Both of these ideas, and their generalizations, are discussed in more detail below.

For the implementation of either approach, some minimal levels of sensing capability are needed onboard the spacecraft. First is the ability to directly sense altitude, either using a laser altimeter or by the efficient processing of stereoscopic optical measurements. This measurement type forms the backbone of an automatic control system to maintain altitude and position relative to an asteroid. In addition to this, it is ideal for the vehicle to have the ability to sense its location relative to the asteroid surface. This can be implemented by optical sensors or scanning lasers. These are not the only types of measurements available or useful, however they are the most essential. The efficient measurement of altitude allows for the implementation of automatic control algorithms that stabilize the spacecraft hovering position, while measurements of body-relative location allows for an expanded capability for the control and motion of the spacecraft. For the latter case, the spacecraft must be able to correlate measured features with a global topography map in order to locate its current location. For some specific applications it may only be necessary to measure and detect lateral motion in addition to vertical motion, however for the most general applications the ability to detemine its global location on the asteroid is necessary. This implies that a global map of the asteroid surface has been created at some point, probably using the same instruments to be used for the relative navigation, although hopefully also using higher resolution scientific instruments. The develpment and implementation of such sensor systems is a technology that is currently being developed, and should be available for use in the future.

In addition to the above sensing and estimation capability, the spacecraft will also require precise 6-DOF control capability. This implies a full set of thrusters for executing arbitrary control moves, perhaps augmented by momentum wheels for fine attitude control. It may be feasible to use more restrictive thruster configurations for the control of the spacecraft, although these would have to be carefully designed for specific implementation approaches. Finally, some, but not all, of these active control approaches imply that the vehicle may be out of sun-light for considerable periods of time. Thus, such power considerations should be factored into the development and design of space vehicles for these advanced approaches.

\section{Near-Inertial Hovering}

In this approach the spacecraft fixes its location relative to the body in the rotating body-sun frame, creating an artificial libration point in this frame. A useful way to think about this approach is to first consider the sun-asteroid libration point. A spacecraft placed in this location will, ideally, remain fixed in its position. If, however, the spacecraft adds a constant thrust acceleration away from the asteroid, it would have to move its location closer to the asteroid in order for the forces to balance again. If a sufficiently large acceleration is added, it could conceivably hold its position relatively close to the asteroid. If it is close to the asteroid, it would have to supply an acceleration of $\sim \mu / r^{2}$ to "hover" at a radius of $r$ from the attracting asteroid. Considering the more general case, it is possible to specify the necessary control thrust to maintain position at an arbitrary location in the asteroid-sun rotating frame. Due to the relatively slow motion of the body about the sun (on the order of degrees per day at fastest), this position can be considered to be nearly inertial over relatively short periods of time. Figure 6 shows a cartoon of how such a maneuver may be implemented.

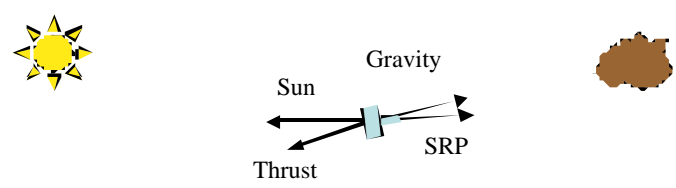

Fig. 6 Near-inertial hovering.

One complication with this approach to hovering is that these artificial equilibrium points are unstable, meaning that a small error in the open-loop thrust acceleration (or a small error in positioning the spacecraft) will cause the spacecraft to depart from its desired hovering point at an exponentially increasing rate. For example, assume a spacecraft is hovering above a spherical asteroid with a constant, open-loop thrust acceleration $a=\mu / R^{2}$, but is positioned at a distance of $R \pm \delta R$ from the asteroid. If the spacecraft is at a slightly higher distance, $R+\delta R$, it's thrust is greater than the gravitational attraction and it will start accelerating away from the asteroid. Conversely, if it is at a slightly lower distance, $R-\delta R$, it's thrust is less than the gravitational attraction and it will start to accelerate towards the asteroid. Thus, practical implementation requires the addition of a closed-loop feedback control that senses the altitude or distance deviation of the spacecraft from its ideal hovering point. The necessary control loop to stabilize this motion is actually quite simple, and can be implemented in an automatic way using minimal spacecraft resources. ${ }^{1}$ There are limits to this approach, however. A spacecraft cannot inertially hover within the maximum radius of the asteroid at its hovering latitude, 
due to obvious physical constraints. Additionally, as the radius of hovering becomes closer to the body, the automatic control approach described here can become unstable, potentially leading to difficulties in implementation.

It is not necessary, however, to force the spacecraft to be fixed precisely at one location. A generalization of this idea places the spacecraft in an elliptic or hyperbolic orbit relative to the asteroid, but has its velocity vector "reflected" whenever it gets within a certain distance to the asteroid (for an elliptic orbit) or gets a certain distance away from the asteroid (for a hyperbolic orbit), forcing the spacecraft to travel back on, or close to, its original path but in the opposite direction (Fig. 7). This approach can be thought of as hovering with a relatively large dead-band control about the nominal hovering point, and requires essentially the same control and sensing capability on-board the spacecraft. This is essentially the approach to be used by the Hayabusa spacecraft when it arrives at its target asteroid. ${ }^{9}$ In this approach the time between control maneuvers can be made arbitrarily long by increasing the size of the dead-band box.

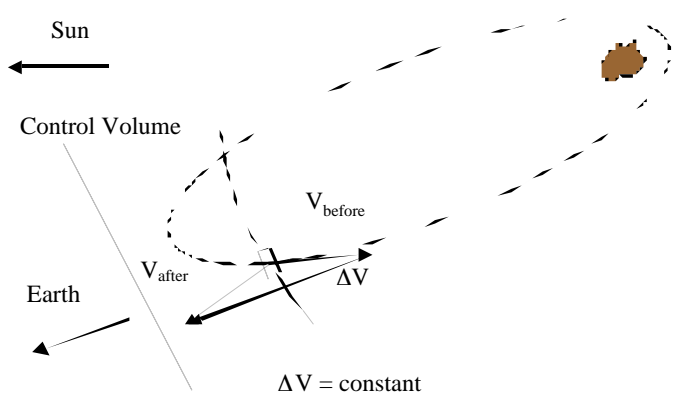

Fig. 7 Practical implementation of a hovering trajectory.

Inertial hovering has several attractive attributes which may make it a mainstay approach of future exploration. There are also a number of drawbacks and limitations, however. On the positive side, this approach can be applied to any small body, and the cost of inertial hovering can theoretically always be driven to zero (not accounting for the statistical control to stabilize the hovering point). However, the position where hovering is feasible may be far from the body, and may not afford the optimal viewing geometry. For example, if the NEAR spacecraft had taken a hovering approach to its mission to Eros and implemented inertial hovering at a distance of $50 \mathrm{~km}$ from the asteroid (which was the nominal orbit radius for most of the mission), it would have required over $15 \mathrm{~m} / \mathrm{s}$ per day to maintain this position, or for its prime 9 month mission would have required a total $\Delta V$ on the order of $4 \mathrm{~km} / \mathrm{s}$. Contrasted with the actual fuel usage (on the order of a few tens of $\mathrm{m} / \mathrm{s}$ ), hovering was clearly not a reasonable approach for that body. Thus, to gain high resolution scientific measurements this approach is largely limited to smaller bodies with their associated smaller hovering cost. A related drawback pertains to the ability of the spacecraft to accurately measure the mass and gravity field of the asteroid, and hence to compile an accurate global topographic map. When using such a controlled hovering mode, errors in the spacecraft thrusters and solar radiation pressure parameters will dominate over the signature of the asteroid gravity field acting on the spacecraft. While it might be possible to extract some averaged results on the total mass of the asteroid, these results would be corrupted by many different uncertainties that will not be uniquely separated from the gravity signature. Additionally, higher order gravity fields will be nearly impossible to extract. This is a serious limitation, as it deprives the mission of essential scientific data and may make it difficult to subsequently transition to a body-fixed hovering exploration of the asteroid. It is possible to enact a few ballistic flybys of the asteroid to gain an improved estimate of its mass, but this estimate will not be as accurate as a mass estimate obtained by tracking the spacecraft while in orbit about the body.

\section{Body-fixed Hovering}

In this approach the spacecraft now fixes its position relative to the rotating body. A natural way to visualize this approach is to imagine using a "jet-pack" to levitate off of the surface of a rotating body, such as the Earth or an asteroid. Since the gravitational attraction is relatively weak at asteroids, it is possible to implement such hovering trajectories for extended periods of time (hours) with total costs on the order of tens of meters per second. This approach to controlling motion in close proximity to small bodies has been analyzed in detail ${ }^{17,20}$ and a detailed simulation of this approach has been developed for analysis of hovering over arbitrary models of asteroids. ${ }^{1}$ Resulting from this work, body-fixed hovering has been shown to be feasible from a dynamics and control perspective. The approach is essentially identical to inertial hovering, except everything is now done relative to the asteroidfixed frame, which generally has a rotation period on the order of hours to days at most. Thus, the spacecraft acceleration must accomodate both the gravitational and centrifugal accelerations, although there are locations where the hovering cost is zero (at the synchronous orbits). This body-fixed hovering approach also suffers from the same basic instability noted for the inertial hovering case, although there are regions where this approach yields completely stabilized motion. ${ }^{20}$ A similar control strategy, using altimetry to maintain a fixed altitude, can stabilize a hovering point so long as it is located within the synchronous radius of the body. This result holds approximately true over the entire body and places an altitude "ceiling" on 
hovering for a simple control law to be able to stabilize its location (see Fig. 8). Some important points relative to this approach can be noted. First is that this technology can be seen as a precursor to a controlled landing on an asteroid surface, and is probably necessary for sample return missions. Second, the Hayabusa spacecraft is, in essence, using this approach when it performs its surface sampling runs. ${ }^{6}$ Third is that this would be a very natural approach to astronaut close proximity operations for human exploration of an asteroid.

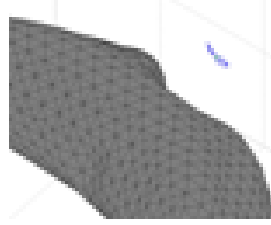

Fig. 8 Body-fixed hovering trajectory.

The implementation of body-fixed hovering can also be accomplished using a simple dead-band controller acting on the altitude of the spacecraft, in conjunction with a single thrust direction properly aligned relative to the asteroid gravity field. Thus, the technology to implement this is clearly available now. If, in addition to maintaining a single altitude at a specific location, it is desired for the spacecraft to translate in the asteroidfixed frame, moving from one site to another, and to descend and ascend from the surface, additional sensing and control technology will have to be used. First, the spacecraft must maintain its attitude in the bodyfixed frame - we should note that it will not do so naturally, as its attitude will remain fixed in inertial space and will want to spin in the asteroid-fixed space. Second, for it to perform translational motions will require that the spacecraft have the capability to locate itself relative to the surface and perform some higherlevel control to null out transverse oscillations about the hovering point.

The development of this surface relative motion capability is perhaps the most advanced concept tendered here. This idea also solves the problem of rover locomotion over an asteroid surface, as instead of relying on natural trajectories induced by mechanical "jumpers", we have controlled motion from one location to another. There are a number of interesting peculiarities associated with such surface relative motion, such as the fact that there is a preferred direction of motion about an asteroid in this mode. ${ }^{17}$ Motion in the same direction as asteroid rotation can actually destabilize the dynamics of the spacecraft control, while translational motion in the opposite direction will tend to stabilize the control system. Other than this observation, which can be easily proven, there is little known about the stability and control of surface relative motion at small bodies, making it an essential topic for future research.

There are a number of drawbacks related to bodyfixed hovering as well. First, it is essential that a fairly accurate model of the asteroid spin, topography and gravity field must be available. The gravity field must be defined down to the surface of the body as well, something which is not always easy to do (as described previously). Thus, body-fixed hovering should be preceeded by a period of characterization at a relatively high level of accuracy. In the future, it may be possible to dispense with this requirement, but that would only be after the basic technology and approach has been proven. Next, there is an altitude limit at which the conventional (and simple) stabilizing control becomes unstable, which could conceivably limit its application, although this does not seem to be that strong of a drawback. Most importantly, however, is that a body-fixed hovering vehicle will undoubtably experience periods of solar occultation, making the presence of batteries or non-solar power generation essential for long-term operations at the surface of a body. Additional operations issues also exist, such as communications, attitude determination, and mechanical interfaces with the surface.

\section{Conclusions}

Ultimately, close proximity missions to hazardous asteroids will be driven by the dynamical environment for the body in question. This is an important point that leads to a strong emphasis on pre-launch and predesign observation campaigns for a mission to such a body. Once the body's dynamical environment has been determined or constrained, there are a number of design approaches that can lead to a successful mission. None of these approaches is without drawbacks, but the wealth of options ensures that there exists a rational and reasonable strategy for exploring and characterizing any small solar system body. This being said, it must also be realized that there is a real diversity among these approaches, and a spacecraft optimized for one specific asteroid mission may not be suitable for a different asteroid.

This is a serious issue for hazardous asteroid mitigation and should be understood by spacecraft and mission designers alike. This fact suggests two different approaches to the problem:

1. The target bodies are sufficiently characterized prior to mission and spacecraft design, allowing for a clearly delineated set of mission operations approaches.

2. The spacecraft and mission is designed to handle a range of possible situations, out of which most asteroids of the class being visited would fall.

The latter approach is clearly more risky as it relies on unknown properties falling within a certain range, 
and in general will lead to a more expensive mission as this fundamental parameter and mission uncertainty must be accounted for at every step in the design. On the plus side, it allows a spacecraft and mission design to be developed prior to the detection of a hazardous asteroid, and should ideally be applicable to a wide range of potential target bodies.

\section{References}

${ }^{1}$ S.B. Broschart and D.J. Scheeres. "Control of hovering spacecraft near small bodies: Application to Asteroid 25143 Itokawa ," Journal of Guidance, Control, and Dynamics, in press.

${ }^{2}$ Dechambre, D. and Scheeres, D.J., (2002). Transformation of spherical harmonic coefficients to ellipsoidal harmonic coefficients, Astronomy and Astrophysics 387: 1114-1122.

${ }^{3}$ Garmier, R., Barriot, J.-P., (2001). Ellipsoidal harmonic expansion of the gravitational potential: Theory and Application. Celestial Mechanics and Dynamical Astronomy 79, 235275.

${ }^{4}$ Hamilton D.P. and Burns J.A. 1991a. Orbital Stability zones about asteroids. Icarus 92, 118-131.

${ }^{5}$ Hamilton D.P. and Burns J.A. 1991b. Orbital Stability zones about asteroids. II. The destabilizing effects of eccentric orbits and of solar radiation. Icarus 96, 43-64.

${ }^{6}$ Hashimoto, T., Kubota, T., Kawaguchi, J., Uo, M., Baba, K. and Yamashita, T., (2001). Autonomous Descent and TouchDown Via Optical Sensors, in Advances in the Astronautical Sciences Vol. 108, 469-480.

${ }^{7}$ Hudson, R.S., Ostro, S.J., Jurgens, R.F., Rosema, K.D., Giorgini, J.D., Winkler, R., Rose, R., Choate, D., Cormier, R.A., Franck, C.R., Frye, R., Howard, D., Kelley, D., Littlefair, R., Slade, M.A., Benner, L.A.M., Thomas, M.L., Mitchell, D.L., Chodas, P.W., Yeomans, D.K., Scheeres, D.J., Palmer, P., Zaitsev, A., Koyama, Y., Nakamura, A., Harris, A. W. and Meshkov, M. N., (2000). Radar Observations and Physical Modeling of Asteroid 6489 Golevka, Icarus 148: 37-51.

${ }^{8}$ Kaula, W.M. 1966. Theory of Satellite Geodesy, Blaisdell.

${ }^{9}$ Kubota, T., Hashimoto, T., Uo, M., Maruya, M. and Baba, K., (2001). Maneuver Strategy for Station Keeping and Global Mapping Around an Asteroid, in Advances in the Astronautical Sciences Vol. 108, 769-780.

${ }^{10}$ Mignard, F. and Hénon, M., (1984). About an unsuspected integrable problem. Celestial Mechanics 33, 239-250.

${ }^{11}$ Miller J.K., Weeks, C. and Wood, L.J., (1990). Journal of Guidance, Control, and Dynamics 13(5) 775-784.

${ }^{12}$ Miller, J.K.,. Bollman, W.E,. Davis, R.P, Helfrich, C.E., Scheeres, D.J., Synnott, S.P., Wang, T.C., Williams, B. and Yeomans, D.K. Navigation Analysis for Eros Rendezvous and Orbital Phases, Journal of the Astronautical Sciences, Vol. 43, 1995, pp. 453 - 476.

${ }^{13}$ Miller, J.K., Konopliv, A. S., Antreasian, P. G., Bordi, J. J., Chesley, S., Helfrich, C. E., Owen, W. M., Scheeres, D. J., Wang, T. C., Williams, B. G. and Yeomans, D. K., (2002). Determination of shape, gravity, and rotational state of Asteroid 433 Eros Icarus 155:3-17.

${ }^{14}$ Morrow, E., Scheeres, D.J. and Lubin, D., (2002). Solar Sail Orbital Operations at Asteroids, Part 2: Exploringthe Coupled Effect of an Imperfectly Reflecting Sail and a Non-Spherical Asteroid, paper presented at the 2002 Astrodynamics Specialist Meeting, Monterrey, California, August 2002. Paper AIAA 2002-4991.

${ }^{15}$ Richter, K. and Keller, H.U., (1995). On the stability of dust particle orbits around cometary nuclei. Icarus 114, 355371.

${ }^{16}$ Sawai, S., Kawaguchi, J., Scheeres, D.J., Yoshizawa, N., Ogawara, M., (2001). Development of a target marker for land- ing on asteroids. Journal of Spacecraft and Rockets 38(4), 601608 .

${ }^{17}$ Sawai, S., Scheeres, D.J. and Broschart, S., (2002). Control of Hovering Spacecraft using Altimetry, Journal of Guidance, Control, and Dynamics 25(4): 786-795.

${ }^{18}$ Scheeres, D.J., (1998). The Restricted Hill Four-Body Problem with Applications to the Earth- Moon-Sun System, Celestial Mechanics and Dynamical Astronomy 70:75-98.

${ }^{19}$ Scheeres, D.J. 1999a. Satellite Dynamics about small bodies: Averaged Solar Radiation Pressure Effects, Journal of the Astronautical Sciences 47:25-46.

${ }^{20}$ Scheeres, D.J., (1999b). Stability of Hovering Orbits around Small Bodies, presented at the 1999 AAS/AIAA Spaceflight Mechanics Meeting, Breckenridge, CO, February 1999. AAS Paper 99-159.

${ }^{21}$ D.J. Scheeres and S. Augenstein. "Spacecraft motion about binary asteroids ," paper presented at the Astrodynamics Specialist Conferences, Big Sky, Montana, August 2003. Paper AAS-03-564.

${ }^{22}$ D.J. Scheeres, "Close Proximity Operations at Small Bodies: Orbiting, Hovering, and Hopping" in Mitigation of Hazardous Comets and Asteroids, M. Belton, T.H. Morgan, N. Samarasinha, D.K. Yeomans, Eds. Cambridge University Press, 2004.

${ }^{23}$ Scheeres, D.J., Ostro, S.J., Hudson, R.S., and Werner, R.A., Orbits Close to Asteroid 4769 Castalia, Icarus, Vol 121, 1996, pp. 67-87.

${ }^{24}$ Scheeres D.J. and Asphaug E.I., (1998). Debris and Sample Transport About Asteroids. In Space 1998 340-346 (R.G. Galloway and S. Lokaj eds.), American Society of Civil Engineering, Reston, Virginia.

${ }^{25}$ Scheeres, D.J., Ostro, S.J. Hudson, R.S., DeJong, E.M. and Suzuki, S. 1998a. Dynamics of orbits close to asteroid 4179 Toutatis. Icarus 132, 53-79.

${ }^{26}$ Scheeres, D.J. and Marzari, F. 2000 Temporary orbital capture of ejecta from comets and asteroids. Astronomy and Astrophysics 356, 747-756.

${ }^{27}$ Scheeres, D.J., Khushalani, B. and Werner, R.A., (2000). Estimating Asteroid Density Distributions from Shape and Gravity Information, Planetary and Space Science 48: 965-971.

${ }^{28}$ Scheeres, D.J. and Marzari, F., (2002). Spacecraft dynamics far from a comet Journal of the Astronautical Sciences Vol. 50 .

${ }^{29}$ Scheeres, D.J., Durda, D.D. and Geissler, P.E. (2002). The Fate of Asteroid Ejecta, in Asteroids III, W.M. Bottke, A. Cellino, P. Paolicchi, and R. P. Binzel, eds., University of Arizona Press, Tucson.

${ }^{30}$ Werner, R.A. and Scheeres, D.J., Exterior gravitation of a polyhedron derived and compared with harmonic and mascon gravitation representations of asteroid 4769 Castalia, Celestial Mechanics and Dynamical Astronomy, Vol. 65, 1997, pp. 313 344 .

${ }^{31}$ Yeomans, D.K., Antreasian, P.G., Barriot, J.-P., Chesley, S.R., Dunham, D.W., Farquhar, R.W., Giorgini, J.D., Helfrich, C.L., Konopliv, A.S., McAdams, J.V., Miller, J.K., Owen Jr., W.M., Scheeres, D.J., Thomas, P.C., Veverka, J., and Williams, B.G.,(2000). Radio Science Results During the NEAR-Shoemaker Spacecraft Rendezvous with Eros, Science 289:2085-2088.

${ }^{32}$ Yoshimitsu, T., Kubota, T., Nakatani, I. and Kawaguchi, J., (2001). Robotic Lander Minerva, Its Mobility and Surface Exploration, in Advances in the Astronautical Sciences Vol. 108, 491-502. 